\title{
Density matrices in $O(N)$ electronic structure calculations: theory and applications
}

\author{
D. R. Bowlert and M. J. Gillant \\ Physics and Astronomy Dept., University College London, Gower Street, London WC1E 6BT, U.K.
}

\begin{abstract}
We analyze the problem of determining the electronic ground state within $O(N)$ schemes, focusing on methods in which the total energy is minimized with respect to the density matrix. We note that in such methods a crucially important constraint is that the density matrix must be idempotent (i.e. its eigenvalues must all be zero or unity). Working within orthogonal tight-binding theory, we analyze two related methods for imposing this constraint: the iterative purification strategy of McWeeny, as modified by Palser and Manolopoulos; and the minimization technique of Li, Nunes and Vanderbilt. Our analysis indicates that the two methods have complementary strengths and weaknesses, and leads us to propose that a hybrid of the two methods should be more effective than either method by itself. This idea is tested by using tight-binding theory to apply the proposed hybrid method to a set of condensed matter systems of increasing difficulty, ranging from bulk crystalline $\mathrm{C}$ and $\mathrm{Si}$ to liquid $\mathrm{Si}$, and the effectiveness of the method is confirmed. The implications of our findings for $O(N)$ implementations of non-orthogonal tight-binding theory and density functional theory are discussed.
\end{abstract}

\section{INTRODUCTION}

The last few years have seen an upsurge of interest in $O(N)$ electronic-structure methods for treating condensed matter both within tight binding theory and within density functional theoryt 22. In these methods, the number of computer operations needed to determine the electronic ground state is proportional to the number $N$ of atoms in the system, instead of showing the $N^{2}$ or $N^{3}$ dependence characteristic of traditional methods. $O(N)$ methods are mossible because electronic phase coherence is localised 12,17,23. This localisation property can be expressed by saying that the density matrix $\rho$ decays to zero with increasing distance.

Some practical $O(N)$ metheds exploit the locality of

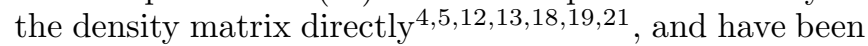
shown to work particularly efficiently for systems with a gap23. They determine the ground state by minimising the total energy with respect to $\rho$, with the approximation that $\rho$ is set equal to zero for spatial separations exceeding some cut-off $R_{\mathrm{c}}$. A central problem in any such approach is that a density matrix must be a projector: it is the operator that projects onto the space of occupied states. Equivalently, one can say that $\rho$ must be idempotent - its eigenvalues must be zero or unity. In practice, the requirement of idempotency is difficult to enforce.

A number of techniques have been suggested for enforcing idempotency 172426 . Many years ago, McWeeny 24 proposed an iterative technique known as 'purification'. The basic idea is that an algorithm is used to transform a nearly idempotent operator $\tilde{\rho}$ into another operator $\rho$ that is even more nearly idempotent. Repetition of this transformation yields a $\rho$ that is idempotent to any desired precision. Later, Li, Nunes and Vanderbilt (LNV) 4 developed a related method using the same transformation, in which the total energy is minimised with respect to $\rho$.

The purpose of this paper is to present arguments for combining these approaches, which we shall refer to as the McWeeny and LNV approaches. We point out that the weaknesses of each approach are matched by the strengths of the other. The fundamental thought here is that McWeeny is good for finding density matrices that are idempotent, while LNV is good for searching through idempotent density matrices to find the one that yields the true ground state. We shall demonstrate that this complementarity can be formulated in a precise and elegant way.

In the next section, we recall the main ideas of the McWeeny and LNV methods for determining the electronic ground state. Section III] is the heart of the paper, where we present our formulation of the complementarity between McWeeny and LNV, and we point out its implications for ground-state search strategy. Practical illustrations of the benefits obtained by combining the two approaches are presented in Sec. IV, and in Secs. V and $\mathrm{VI}$ we give discussion and conclusions.

\section{THE MCWEENY AND LNV METHODS}

The arguments we shall develop are very general, but for simplicity we present them in the framework of orthogonal tight-binding theory. We comment later on the extensions to the non-orthogonal case and to density functional theory.

In the orthogonal tight-binding basis, the matrix elements of the Hamiltonian are denoted by $H_{i j}$, where $i$ and $j$ go over all basis functions on all atoms $(1 \leq i, j \leq$ $N_{b}$, where $N_{b}$ is the total number of basis functions in the system). If there are $N_{0}$ occupied states, then the ground-state energy $E_{0}$ is given by: 


$$
E_{0}=\min \operatorname{Tr}(H \rho),
$$

where the minimisation is performed with respect to all Hermitian matrices $\rho$, subject to the conditions that $\rho$ is a projector $\left(\rho^{2}=\rho\right)$ and that $\operatorname{Tr} \rho=N_{0}$. Note that the factor of two due to spin is omitted for simplicity. The ground-state density matrix is given by:

$$
\rho=\theta(\mu I-H),
$$

where $\theta(x)$ is the Heaviside function $(\theta=1$ for $x>0$ and $\theta=0$ for $x<0$ ) and $\mu$ is the chemical potential (Fermi energy). Instead of working at fixed number of occupied states, it is sometimes more convenient to work at fixed chemical potential, in which case we minimise the grand potential $\Omega$ :

$$
\Omega_{0}=\min \operatorname{Tr}(H-\mu I) \rho
$$

subject only to the condition $\rho^{2}=\rho$.

If one was allowed to diagonalise $H$, then $\rho$ could be straightforwardly expressed as:

$$
\rho_{i j}=\sum_{n=1}^{N_{0}} c_{i n} c_{j n}^{*},
$$

where $c_{i n}$ are the eigenvector components of $H$ :

$$
\sum_{j=1}^{N_{b}} H_{i j} c_{j n}=\epsilon_{n} c_{i n},
$$

and the eigenvalues $\epsilon_{n}$ are assumed to be in ascending order. But diagonalisation is an $O\left(N^{3}\right)$ process and is incompatible with linear scaling.

\section{A. McWeeny purification}

The McWeeny purification scheme 24 gives a way of achieving idempotency without diagonalisation. The purification algorithm for mapping a nearly idempotent matrix $\tilde{\rho}$ into one that is more nearly idempotent is:

$$
\rho=3 \tilde{\rho}^{2}-2 \tilde{\rho}^{3} .
$$

The way this mapping works can be understood by considering the eigenvalues of $\rho$ and $\tilde{\rho}$, denoted by $\lambda, \tilde{\lambda}$ respectively. Since $\rho$ is diagonal in any representation that diagonalises $\tilde{\rho}$, the relationship between their eigenvalues is:

$$
\lambda=3 \tilde{\lambda}^{2}-2 \tilde{\lambda}^{3} .
$$

From the form of the function $f(\tilde{\lambda})=3 \tilde{\lambda}^{2}-2 \tilde{\lambda}^{3}$ (see Figure (1), it follows that if $-\frac{1}{2}<\tilde{\lambda}<\frac{3}{2}$ then $0 \leq \lambda \leq 1$. Furthermore if $0<\tilde{\lambda}<1$ then for $\tilde{\lambda}<\frac{1}{2}, \lambda<\tilde{\lambda}$ and for $\tilde{\lambda}>\frac{1}{2}, \lambda>\tilde{\lambda}$, so that iteration of the mapping drives the eigenvalues towards the values 0 or 1 .
As this process continues, the final approach to idempotency accelerates rapidly. If $\tilde{\lambda}$ deviates from zero by a small amount, then the deviation of $\lambda$ is proportional to the square of that amount, and similarly for deviations from unity. This quadratic convergence to idempotency can be summarised by noting that the matrices $\rho^{2}-\rho$ and $\tilde{\rho}^{2}-\tilde{\rho}$ are related by:

$$
\rho^{2}-\rho=4\left(\tilde{\rho}^{2}-\tilde{\rho}\right)^{3}-3\left(\tilde{\rho}^{2}-\tilde{\rho}\right)^{2} .
$$

For a given initial matrix $\rho^{(0)}$, iteration of the purification algorithm therefore generates a sequence $\rho^{(1)}, \rho^{(2)}, \ldots$ that converges to an idempotent matrix $\rho^{(\infty)}$. However, there are many idempotent matrices, and $\rho^{(\infty)}$ is not necessarily the idempotent matrix given by eqn (2). The latter is uniquely specified by the statements that (a) $\rho$ commutes with $H$; (b) in a representation that diagonalises $\rho$ and $H$, the eigenvalues $\lambda_{n}$ of $\rho$ (which by idempotency must be 0 or 1) are given by $\lambda_{n}=1$ for $\epsilon_{n}<\mu$ and $\lambda_{n}=0$ for $\epsilon_{n}>\mu$.

It has been emphasised recently by Palser and Manolopoulos 26 (hereafter PM) that McWeeny purification automatically delivers the correct ground state provided the initial $\rho$ is an appropriate function of the Hamiltonian whose eigenvalues are in the range $(0,1)$. The initial $\rho$ then commutes with $H$, and this means that all subsequent $\rho^{(k)}$ commute with $H$. Provided the eigenvalues of $\rho^{(0)}$ satisfy $0<\lambda_{n}<\frac{1}{2}$ for $\epsilon_{n}>\mu$ and $1>\lambda_{n}>\frac{1}{2}$ for $\epsilon_{n}<\mu$, then repeated purification automatically yields a final $\rho$ representing the exact ground state. PM point out that $\rho^{(0)}$ satisfies the requirements if it is chosen as:

$$
\rho^{(0)}=\frac{1}{2} \xi(\mu I-H)+\frac{1}{2} I,
$$

where

$$
\xi=\min \left\{\frac{1}{H_{\max }-\mu}, \frac{1}{\mu-H_{\min }}\right\},
$$

with $H_{\min }, H_{\max }$ lower and upper bounds on the eigenvalue spectrum of $H$. A crucial feature of this procedure is that the grand potential $\Omega^{(n)}$ converges monotonically to the ground state value from above: $\Omega^{(n+1)}<\Omega^{(n)}$. PM further show that a purification procedure working at fixed $N_{0}$ can be obtained with a modified version of the McWeeny algorithm; details are given in their paper. In this case, the energy $E^{(n)}$ converges monotonically from above: $E^{(n+1)}<E^{(n)}$.

The McWeeny purification scheme, as modified by PM, should therefore provide an extremely effective method for determining the ground state, provided everything is done exactly. But the essence of $O(N)$ methods is the imposition of a spatial cut-off on the density matrix. This means that at each purification step the input matrix $\tilde{\rho}_{i j}$ will be non-zero only if $j$ is one of a spatially localised set of neighbours of $i$. Because of the matrix multiplications, the output matrix $\rho_{i j}$ will have a more extended range, 
so that it must be truncated back to the imposed range before each iteration. Because of this truncation, the monotonic convergence of $\Omega$ or $E$ must fail as the ground state is approached, and PM suggest that this failure of monotonicity be used as a criterion for terminating the iterative process. In other words, should the energy for any given iteration be higher than that from the previous iteration, the process should be stopped. This is an important criterion to follow, as once the truncation errors become significant, the iteration will no longer converge on an idempotent matrix.

This heuristic criterion for terminating the iterations may work in some cases, but we consider it to be unsatisfactory for two reasons. First, it makes the approximate ground-state energy depend on the details of the initial $\rho^{(0)}$. Second, the energy is not the minimum of any function, so that the variational property of the exact ground state is lost. This means that calculated forces on atoms will not be consistent with the energy, so that both relaxation to equilibrium and dynamics are likely to be problematic. In addition, the use of this procedure as part of an $O(N)$ density functional scheme would encounter other problems, as we point out in Sec. V.

\section{B. LNV minimisation}

The scheme of Li, Nunes and Vanderbiltt also makes use of the purification algorithm, but in a completely different way. If we work at constant $\mu$, then the strategy is based on minimisation of $\Omega=\operatorname{Tr}(H-\mu I) \rho$, subject to the condition that $\rho$ is weakly idempotent (this means that its eigenvalues $\lambda$ satisfy $0 \leq \lambda \leq 1)$. In this domain of matrices, the minimum value of $\Omega$ is obtained when $\rho$ is given by eqn (2). This is easily seen by writing $\Omega$ in a representation in which $H$ is diagonal (but $\rho$ is not assumed to be diagonal):

$$
\Omega=\sum_{n}\left(\epsilon_{n}-\mu\right) \rho_{n n} .
$$

The diagonal elements of a weakly idempotent matrix must lie in the range $[0,1]$, so that the minimum of $\Omega$ is obtained when $\rho_{n n}=1$ for $\epsilon_{n}<\mu$ and $\rho_{n n}=0$ for $\epsilon_{n}>\mu$. In this case, it is readily shown that all the offdiagonal elements of $\rho_{n n}$ must vanish, and $\rho$ is given by eqn (2).

The constraint of weak idempotency can be achieved by expressing $\rho$ as in eqn (6), provided the eigenvalues of $\tilde{\rho}$ lie in the range $\left(-\frac{1}{2}, \frac{3}{2}\right)$. In this role, $\tilde{\rho}$ is simply an auxiliary matrix, introduced solely to satisfy weak idempotency. Then $\Omega$ can be written as:

$$
\Omega=\operatorname{Tr}(H-\mu I)\left(3 \tilde{\rho}^{2}-2 \tilde{\rho}^{3}\right) .
$$

But since this is a cubic form in $\tilde{\rho}$, it can have only a single minimum, which is obtained when $\tilde{\rho}=\rho=\theta(\mu I-H)$. In practice, it is often more convenient to minimise the energy $E=\operatorname{Tr} H\left(3 \tilde{\rho}^{2}-2 \tilde{\rho}^{3}\right)$ subject to the constraint that the number of occupied states $N_{0}=\operatorname{Tr}\left(3 \tilde{\rho}^{2}-2 \tilde{\rho}^{3}\right)$ is held constant. This requires more computational effort, since the gradient of the electron number with respect to the density matrix must be evaluated; there are severalefficient implementations of the constant- $N_{0}$ constraint 2728.

The great advantage of LNV over McWeeny is that it is variational, and this means that it works even when a spatial cut-off is imposed on $\tilde{\rho}$. Indeed, it is one of the standard methods of achieving linear-scaling behaviour in tight-binding calculations23. With a cut-off, the minimum of $\Omega$ or $E$ is guaranteed to be above the exact ground-state value, and this minimum decreases monotonically to the exact value as the cut-off is increased. The variational property means that forces on the atoms calculated at the minimum are exactly consistent with the variations of $\Omega$ or $E$.

Nevertheless, the LNV techique does have several weaknesses, all of which affect the process of searching for the ground state. An obvious weakness is that it does not have the quadratic convergence shown by the McWeeny technique. Any iterative method used to minimise $\Omega$ or $E$ will give linear convergence, so that as we approach the minimum the error in $\tilde{\rho}$ at each iteration is some fraction of the error at the previous iteration. This means that - at least in the absence of a spatial cut-off LNV is expected to need more iterations than McWeeny to achieve a given accuracy.

This weakness is exacerbated by the fact that the LNV technique demands more operations in each iteration, as has been emphasised by PM26. This is simply because we need to calculate the gradient of $\Omega$ or $E$ with respect to the elements of $\tilde{\rho}$, in addition to calculating $\Omega$ or $E$ itself. So even if it did not need more iterations, LNV would still be slower. Roughly speaking, an LNV iteration takes about twice as long as a McWeeny iteration in constant- $N_{0}$ calculations and somewhat more than this in constant- $\mu$ calculations.

There is also a third cause of slowness in LNV, namely ill conditioning. In any minimisation problem, convergence to the minimum will be slow if the curvatures of the function are very different in different directions, or equivalently if the eigenvalues of the Hessian matrix span a wide range. But it is readily shown that the curvatures of $\Omega$ are determined by the quantities $\left|\epsilon_{n}-\mu\right|$, which will indeed span a wide range unless the system has a large band-gap. We should expect this weakness to be particularly troublesome for metallic systems, and in fact detailed evidence for the inefficiepcy of LNV for such systems has already been presented23. The McWeeny technique does not suffer from this problem.

In addition to these problems of convergence speed, LNV has two other obstacles: initialisation and poor robustness. It is far from clear how best to initialise the density matrix within LNV; in general, the ansatz of $\tilde{\rho}=\frac{1}{2} \mathbf{I}$ is made within orthogonal tight binding 127 . Poor robustness arises since $\Omega$ is a cubic form in $\tilde{\rho}$ and is unbounded below. In order for a minimisation method to 
lead to the minimum of $\Omega$, the initial $\tilde{\rho}$ must be chosen close enough to the minimum. If we start from an unsuitable initial $\tilde{\rho}$, any downhill search method will lead away from the minimum, and $\Omega$ will plunge towards infinitely negative values. In a robust search strategy we expect to seek the minimum of $\Omega$ in a sequence of search directions. It is a sign of danger if $\Omega$ has a point of inflection but no minimum in a search direction, and we shall use this idea later when discussing robustness.

We show in the next section how a combination of McWeeny and LNV allows their strengths to be exploited and their weaknesses to be avoided.

\section{RELATIONSHIP BETWEEN MCWEENY AND LNV}

In analysing the relationship between the two methods, we find it helpful to regard matrices as elements of a vector space. Using this viewpoint, we shall make free use of geometrical concepts such as the straight line joining two matrices $A$ and $B$, by which we mean the set of matrices $(1-\lambda) A+\lambda B$, where $0<\lambda<1$. We define the magnitude or norm of a matrix $A$ as:

$$
\|A\|=\left[\operatorname{Tr}\left(A^{\dagger} A\right)\right]^{1 / 2},
$$

where $A^{\dagger}$ is the Hermitian conjugate of $A$. This allows us to talk of the 'distance' $\|A-B\|$ between two matrices. We shall also need the notion of scalar product of two matrices, defined as:

$$
(A, B)=\operatorname{Tr}\left(A^{\dagger} B\right) .
$$

Matrices are referred to as 'orthogonal' if $(A, B)=0$.

In the vector space just defined, there is a manifold consisting of all matrices that are idempotent, and we call this the idempotency surface. For any suitably chosen matrix $A^{(0)}$, the purification algorithm (Eq. (6)) generates a sequence of matrices $A^{(1)}, A^{(2)}, \ldots$ which tend to a limit $A^{(\infty)}$ lying on the idempotency surface. If we imagine this sequence of points in the vector space as joined by straight lines, then we form a path which we refer to as the McWeeny path.

The following two statements, proved in Appendix A, will play a key role in our proposed strategy for finding the ground state:

1. All McWeeny paths meet the idempotency surface orthogonally;

2. For any point on the idempotency surface, the gradient of the LNV function is tangential to the surface.

A picture illustrating these statements is shown in Fig. 22.

To explain more precisely what these statements mean, we need to define the concept of tangent planes to the idempotency surface. For any point $P$ on this surface
$\left(P^{2}=P\right)$, consider points on the straight line $\tilde{P}=P+$ $\alpha B$, where $B$ is some chosen Hermitian matrix and $\alpha$ is a real scalar variable. In general, $\tilde{P}$ will not be idempotent, but for some choices of $B, \tilde{P}$ is idempotent to linear order in $\alpha$ :

$$
\tilde{P}^{2}-\tilde{P}=\alpha^{2} B^{2}
$$

For such choices of $B$, the shortest distance between a given point on the straight line and the idempotency surface is of order $\alpha^{2}$, and we can say that the straight line is a tangent line to the idempotency surface. The tangent plane at the point $P$ consists of all matrices $\tilde{P}$ on all tangent lines passing through $P$. A convenient way to construct points in the tangent plane is described in Appendix A.

Now suppose we have a McWeeny sequence $A^{(k)}$ going to the limit $A^{(\infty)}$. Then the meaning of statement (1) is that in the $k \rightarrow \infty$ limit the difference vector $A^{(k)}-A^{(\infty)}$ becomes orthogonal to every vector $B$ for which $A^{(\infty)}+B$ is in the tangent plane passing through $A^{(\infty)}$ :

$$
\lim _{k \rightarrow \infty}\left(B, A^{(k)}-A^{(\infty)}\right) /\left\|A^{(k)}-A^{(\infty)}\right\|=0 .
$$

The meaning of statement (2) is that, if $F$ is the gradient of the LNV function at point $P$ on the idempotency surface, then $P+\alpha F$ is in the tangent plane passing through $P$. The corollary is that for idempotent $P$ the LNV gradient is orthogonal to the McWeeny path.

Consider the implications of the two statements. We know that purification is a completely robust way of reaching idempotency. Statement (1) implies that it is also very direct. As we approach the idempotency surface, we are following the shortest possible path. Once we are near the surface, purification is tantamount to dropping a perpendicular onto the surface. In addition, quadratic convergence means that the approach to the surface accelerates rapidly in the final stages. But for a general starting point purification does not give us the ground state. It gives us an idempotent density matrix, but not the idempotent density matrix corresponding to the ground state. This is where LNV comes in. Once we are on the idempotency surface, the two statements guarantee that application of LNV does not undo what was achieved by McWeeny, because it keeps us on the surface to first order. Furthermore, LNV is a completely robust way of finding the ground state if we are constrained to the surface. Within the idempotency constraint, the LNV function has only a single minimum, and cannot fall below the ground state energy.

We have assumed up to now that everything is done exactly, without any spatial cut-off. In this case, purification by itself is enough to find the ground state. As stressed by PM (see Sec. II ), an initial guess for $\rho^{(0)}$ given by eqns (9) and (10) guarantees that repeated purification delivers the unique density matrix corresponding to the ground state. But with a cut-off the situation is quite different. Purification then brings us near to the ground 
state, but leaves us with a ground-state estimate lacking variational properties. At this point, we suggest that the effective strategy is to switch to LNV. Since we are already near the ground state, minimisation of the LNV function should be rapid. Although we are not exactly on the idempotency surface, the energy gradient should still maintain idempotency to good accuracy, and there should be no danger of approaching the unstable region where the LNV function decreases unboundedly.

An important benefit of combining McWeeny and LNV in the way we suggest is that it makes it easier to work at constant electron number, which is usually what one wishes to do. Although the modifications proposed by PM make it straightforward to perform McWeeny purification at constant $N_{0}$, the minimization of the LNV function is made more complicated by the need to hold $N_{0}$ fixed. But once we are on the idempotency surface, this difficulty in the LNV scheme disappears. This can be seen by recalling that $N_{0}=\operatorname{Tr}\left[3 \tilde{\rho}^{2}-2 \tilde{\rho}^{3}\right]$, so that $\partial N_{0} / \partial \tilde{\rho}_{i j}=6\left(\tilde{\rho}-\tilde{\rho}^{2}\right)_{i j}$, which vanishes if $\tilde{\rho}$ is idempotent. The implication is that, since the LNV gradient keeps $\tilde{\rho}$ near the idempotency surface once it has been brought there by McWeeny purification, the electron number will automatically maintain itself almost constant during the LNV stage. Practical tests of this will be shown in the next section.

In summary, the proposed hybrid strategy capitalizes on the expected robustness and speed of purification, but avoids its lack of variational properties. Variational behaviour is supplied by the LNV component of the strategy, but since this is used only in the final stages, we hope to avoid the instability and slowness from which LNV can suffer if used alone. An important feature to note is that, as the cut-off radius increases, we expect the hybrid strategy to become increasingly effective: almost all the work is then done by purification, so that the strengths of McWeeny and LNV should complement each other better and better.

\section{PRACTICAL EXAMPLES}

In this section we present examples of calculations done using the proposed hybrid approach, compared with pure LNV calculations; these examples form a sequence of systems of increasing difficulty: bulk, diamond-structure carbon and silicon; a relaxed carbon vacancy; the $\mathrm{Si}(001)$ surface; and finally liquid silicon.

There are two main characteristics that we are looking for in these tests: speed and robustness. The speed of a method can be gauged either by the total number of iterations taken, or by the total CPU time used. These give different measures, since each McWeeny iteration requires only about half as much time as an LNV iteration. When comparing different methods, we shall characterize the speed by the number of iterations needed to reach the ground state within a specified tolerance, and the implications for CPU time will be pointed out where appropriate.

Robustness is harder to characterize. As explained in Sec. IIB, the LNV method can become unstable if the grand potential $\Omega$ has no minimum in a search direction, because this signals that we are approaching a dangerous region where $\Omega$ decreases unboundedly. This behaviour is, indeed, found on occasions in calculations based on pure LNV, particularly when the electron number is kept constant (as opposed to the electron chemical potential). The kind of robustness we are looking for in our hybrid method is therefore the absence of this kind of instability. Rather than repeat the refrain throughout each section, we will state now that we have not seen this behaviour at any time during these simulations.

To perform the tests, we have used a simple, nearestneighbour orthogonal tight binding model, with parameterisations for silicon 30 and carbon 31 . We adapted an implementation of the LNV scheme by Goringe27. In this scheme, the density matrix cut-off is not defined as a sphere, but rather in terms of a cluster of atoms. This cluster is formed by including all atoms which are within range of a certain number of 'hops' of the central atom (e.g. a nearest neighbour is at one hop, and its nearest neighbours are at two hops from the central atom). The localisation criterion is thus specified in terms of the number of hops. All McWeeny minimisations have been carried out at fixed electron number; except where stated, the same is true for LNV minimisations and LNV stages of hybrid minimisations.

The initial density matrix for the pure LNV method was chosen to be $\frac{1}{2} \mathbf{I}$, while for the hybrid and McWeeny methods it was constructed according to equations (9) and (10). When using the hybrid method, the switch to the LNV phase from the McWeeny phase occurred when the energy in one iteration was higher than that in the previous iteration (which is indicative of truncation error) or an equivalent error was found in the maintenance of electron number (as described by Palser and Manolopoulos 26). As the density matrix had been shown to be invalid, the density matrix from the previous iteration was passed to the LNV phase as an initial matrix.

\section{A. Perfect Si and C crystals}

We have used our proposed hybrid scheme and the pure LNV scheme to find the electronic ground state for carbon and silicon in the diamond crystal structure for different cut-off radii of the density matrix $(3,5$ and 7 hops). Tables 1 and II give the number of iterations taken by the two methods to achieve a specified tolerance in fractional change in cohesive energy between line minimisations (in this case, $10^{-8}$ ). This is a standard type of criterion applied in practical minimisation, and is used here to show the performance of the methods in practical tests; in both cases, the methods are achieving the ground 
state, which ensures that a fair comparison is made. For the hybrid scheme, we give separately the numbers of iterations needed in the McWeeny and LNV stages of the calculation, while for the LNV scheme we simply give the total number of iterations. As described above in sections II A and II B, the initialisations used are the PME6 $\rho^{(0)}$ for the hybrid initialisation and $\frac{1}{2} \mathbf{I}$ for the LNV initialisation. Several points should be noted. First, the total numbers of iterations are the essentially the same for both schemes. As pointed out above, this means that the hybrid scheme is significantly faster (by about 25 $\%$ ). Second, the LNV stage of the hybrid scheme takes fewer iterations as the cut-off radius is extended. This is expected, because the McWeeny stage should bring the density matrix closer to the ground state for larger radii. To confirm this point, we have calculated the norm (see Eq. 13) of the difference between the density matrix obtained at the end of the McWeeny stage and final ground-state density matrix. This norm, reported in the last column of Tables If and II, decreases markedly with increasing cut-off radius. Finally, it is worth noting that the $\mathrm{Si}$ crystal takes somewhat more iterations that the $\mathrm{C}$ crystal, as might be expected because of its smaller band gap.

The way in which the energy converges to its groundstate value in the pure LNV scheme and in the LNV stage of the hybrid scheme is shown in Figures 3 and 4 , where we report the difference between the cohesive energy at each iteration and the final ground-state cohesive energy, as a fraction of the final cohesive energy. The results show very clearly that the McWeeny stage gets closer and closer to the correct ground state as the cutoff radius increases. The rate of convergence in the LNV stage is essentially the same as that found in the pure LNV scheme, as expected. In all cases, the error in the energy decrease approximately exponentially with iteration number.

\section{B. Carbon Vacancy}

When a vacancy is introduced into diamond-structure carbon, the degeneracy of the dangling-bond states is broken by a Jahn-Teller distortion which lowers the symmetry of the system, and defect states appear in the band gap. This system therefore gives us an interesting increase in complexity compared with the perfect crystal.

Table III reports the numbers of iterations taken by the hybrid and pure LNV schemes. The behaviour is similar to that found for the perfect crystal, although for small cut-off radii the total number of iterations in the hybrid scheme is now slightly larger than for pure LNV. Nevertheless, the hybrid scheme is still significantly faster than pure LNV in terms of CPU time. For the present system, we have examined the consequences of working at constant $\mu$ rather than constant electron number in the LNV stage of the hybrid scheme. To do this, we have fixed $\mu$ at the value of $\nabla N_{o} \cdot \nabla E / \nabla N_{o} \cdot \nabla N_{o}$, which is the correct definition of the chemical potential for electrons. The final column of Table III shows the deviation of total electron number from its nominal value of 252 (the calculation was done with a system of 63 atoms) in the final ground state when the calculation is done like this. The very small deviations, which decrease with increasing cut-off radius, confirm our expectation that $N_{0}$ automatically holds itself almost constant in the LNV stage (see Sec. III).

\section{C. $\operatorname{Si}(001)$ surface}

The $\mathrm{Si}(001)$ surface is a complex electronic system. Rebonding between surface atoms causes strong displacements from perfect-lattice positions and the formation of dimers, which themselves become buckled because of Jahn-Teller distortion29. These effects give rise to bands of gap states. Table IV shows the numbers of iterations required by the hybrid and pure LNV schemes. It is clear that in this case the hybrid scheme is significantly faster than pure LNV, by a factor of between 3 and 5 . We believe that the slowness of pure LNV is a manifestation of ill conditioning caused by gap states (see Sec. II B), which do not appear to affect the McWeeny stage of the hybrid method. As in the $\mathrm{C}$ vacancy case, we have examined the deviations of electron number caused by running the LNV stage of the hybrid method in constant- $\mu$ mode, and these are reported in the final column of Table IV. Once again, they are very small, though not quite so small as in the vacancy case.

\section{Liquid silicon}

Liquid Si is a challenging system for any method, since it is both disordered and metallic. But the important point here is that dynamical simulation must be performed, so that energy conservation is important, and this means that the consistency of energy and ionic forces is crucial. We have pointed out in Sec. III that this consistency is ensured by the variational property of both pure LNV and our hybrid method. By contrast, pure McWeeny is not variational, so we are particularly interested to find out the consequences of attempting to use pure McWeeny for this system.

The simulations were done using a repeating cell of 64 atoms at an initial temperature of $3000 \mathrm{~K}$. As before, the orthogonal tight-binding model described in Ref. 30 was used. An important technical point for the pure LNV simulations is that the changes in bonding from one step to the next are so strong that it is not helpful to use the final density matrix from one step as the initial guess for the density matrix at the next step 32 . Instead, the standard initialisation of $\frac{1}{2} \mathbf{I}$ is used. It must be stressed that because of the discontinuous changes of bonding caused 
by the spatial cut-off of the density matrix, energy conservation will be far from perfect whatever method is used. The point at issue is therefore the relative quality of energy conservation for different methods.

Another relevant technical point concerns the occupation numbers to be used for the electronic states. There would be sound physical arguments for using Fermi-Dirac occupation numbers corresponding to the temperature of the simulation. For the present tests, we have instead set the electronic temperature to zero, so that the occupation numbers are exactly unity below the Fermi energy and exactly zero above, following the theory of previous Sections. The motivation for doing this is that it provides a more stringent test of $O(N)$ methods for metallic systems, since increase of the electronic temperature has the effect of localizing the density matrix, as described in Ref. 23.

We have performed our $l$-Si simulations in all three possible ways: pure LNV, hybrid, and pure McWeeny, using a spatial cut-off of 3 hops on the density matrix. We find that the hybrid method is once again faster than pure LNV: on average, the hybrid calculations require 13 McWeeny and 13 LNV iterations per step, while pure LNV requires 26 iterations, so that the hybrid method is between $25 \%$ and $50 \%$ faster than pure LNV.

Fig. 5 shows the results of our tests on energy conservation over a period of $0.1 \mathrm{ps}$. The variation of the total energy is almost exactly the same in the LNV and hybrid methods and consists of a steady upward drift of $\sim 0.03 \mathrm{eV} /$ atom during the $0.1 \mathrm{ps}$ period. This is already large, since it corresponds to a temperature increase of ca. $100 \mathrm{~K}$. But with pure McWeeny, the increase of total energy is about 25 times larger, and in our judgment this does not give a satisfactory method of doing dynamics for this kind of system. We regard this as a compelling reason for not using McWeeny by itself.

\section{DISCUSSION}

The theoretical arguments presented in Secs. II and III showed that the McWeeny and LNV techniques behave in very different ways in the search for the ground state, and indicated that a combination of the two techniques should be more efficient and robust than either technique by itself. The practical tests we have just reported fully support these ideas. For the five systems we have studied, they confirm that in terms of CPU time the hybrid technique is always faster, and sometimes much faster, than the LNV technique by itself. They also confirm that the hybrid technique is more robust, since the LNV iterations used in the final stage show no sign of the unstable behaviour that can occur if LNV is used thoughout. We have seen that McWeeny used by itself, following the proposals of Palser and Manolopoulos 26 , can indeed produce very accurate results for the ground-state energy, especially when the spatial cut-off is increased, but that its lack of consistency between energy and ionic forces can cause serious problems in dynamical simulations. Importantly, our results also demonstrate that the hybrid method allows one to work at constant electron number with greater ease than when LNV is used by itself.

Although we have chosen to work within orthogonal tight-binding theory, the main ideas carry over directly to the non-orthogonal case. Nunes and Vanderbilt 34 have already shown how to generalize the LNV scheme to perform $O(N)$ tight-binding calculations with nonorthogonal orbitals, and Palser and Manolopoulos have shown that McWeeny purification can be generalized in the same way. We demonstrate in Appendix B that our two key statements presented in Sec. III remain valid in the non-orthogonal case, provided the scalar product between matrices is defined using the appropriate metric. The discussion in Appendix B indicates that the only significant problem in using our hybrid $O(N)$ scheme for doing practical calculations within non-orthogonal tightbinding theory is that one needs the inverse of the overlap matrix $S_{i j}$ between orbitals. However, we believe that an approximate inverse of $S_{i j}$ should suffice, and ways of obtaining a suitable approximation have been proposed by Palser and Manolopoulos26. 33.

The ideas we have presented should also beapplicable to $O(N)$ DFT. In a series of recent papers $13,18,19,22$ we have shown how a practical $O(N)$ DFT scheme can be constructed, the guiding principle being the minimization of the DFT total energy with respect to the density matrix. The ideas have been implemented in our code CONQUEST19 (Concurrent $O(N)$ QUantum Electronic Structure Technique), in which the density matrix is represented in a basis of localized orbitals which are varied to minimize the total energy. In the present form of CONQUEST, the minimization is performed in three nested loops. In the innermost loop, the energy is minimized wtih respect to the elements of the density matrix, and the two outer loops have the tasks of achieving self-consistency and minimizing with respect to the localized orbitals. The operations performed in the innermost loop are therefore identical to those performed in non-orthogonal tight-binding theory, and the methods we have presented here should be applicable without change to this part of $O(N)$ DFT.

The relevance of the present ideas to $O(N)$ DFT gives another reason why the LNV component of our hybrid scheme is so important. In order to minimize the total energy with respect to the localized orbitals, it is essential to have an analytic expression for the gradient of the energy with respect to variations of these orbitals. But the existence of such an expression for the gradient relies crucially on the energy being stationary with respect to variations of the density matrix. So once again the variational behaviour given by LNV is essential, just as it is for calculating forces - and for basically the same reason. We are currently studying the improvements that can be achieved in $O(N)$ DFT by applying the ideas we have presented. 


\section{CONCLUSIONS}

We have shown that a combination of McWeeny purification and LNV minimization methods gives a robust and rapid means of searching for the electronic ground state in the framework of an $O(N)$ density-matrix scheme. The McWeeny stage finds an idempotent density matrix quickly and efficiently, and the LNV stage then finds the idempotent density matrix which minimises the total energy. We have presented examples which demonstrate the advantages of this hybrid scheme, and shown why both stages are necessary. We have pointed out that the main ideas can be generalized to non-orthogonal tight-binding theory and $O(N)$ density functional theory.

\section{ACKNOWLEDGMENTS}

We are happy to acknowledge useful discussions with David Manolopoulos and the early viewing of Ref. 26. We would also like to acknowledge useful discussions with Chris Goringe, and the use of his density matrix code, DensEl. The work of DRB and the computer facilities used in the calculations are supported by EPSRC grant GR/M01753. The work of MJG is financially supported by CCLRC and GEC.

* Electronic address: david.bowler@ucl.ac.uk

$\dagger$ Also at DCI, CLRC Daresbury Laboratory, Warrington, WA4 4AD. Electronic address: m.gillan@ucl.ac.uk

${ }^{1}$ D. G. Pettifor, Phys. Rev. Lett. 63, 2480 (1989).

${ }^{2}$ W.Yang, Phys. Rev. Lett. 66, 1438 (1991).

${ }^{3}$ G. Galli and M. Parrinello, Phys. Rev. Lett. 69, 3547 (1992).

${ }^{4}$ X.-P. Li, R. W. Nunes and D. Vanderbilt, Phys. Rev. B 47, 10891 (1993).

${ }^{5}$ M. S. Daw, Phys. Rev. B 47, 10895 (1993)

${ }^{6}$ P. Ordejón, D. Drabold, M. Grumbach and R. Martin, Phys. Rev. B 48, 14646 (1993).

${ }^{7}$ M. Aoki, Phys. Rev. Lett. 71, 3842 (1993).

${ }^{8}$ F. Mauri, G. Galli and R. Car, Phys. Rev. B 47, 9973 (1993).

${ }^{9}$ S. Goedecker and L. Colombo, Phys. Rev. Lett. 73, 122 (1994).

${ }^{10}$ E. B. Stechel, A. R. Williams and P. J. Feibelman, Phys. Rev. B 49, 10088 (1994).

${ }^{11}$ W. Hierse and E. B. Stechel, Phys. Rev. B 50, 17811 (1994).

${ }^{12}$ W. Kohn, Int. J. Quant. Chem. 56, 229 (1995).

${ }^{13}$ E. Hernández and M. J. Gillan, Phys. Rev. B 51, 10157 (1995).

14 J. D. Kress and A. F. Voter, Phys. Rev. B 53, 12733 (1996).

15 A. P. Horsfield, Mat. Sci. Engin. B 37, 219 (1996).
16 A. P. Horsfield, A. M. Bratkovsky, M. Fearn, D. G. Pettifor and M. Aoki, Phys. Rev. B 53, 12964 (1996).

${ }^{17}$ W. Kohn, Phys. Rev. Lett. 76, 3168 (1996).

${ }^{18}$ E. Hernández, M. J. Gillan and C. M. Goringe, Phys. Rev. B 53, 7147 (1996).

${ }^{19}$ C. M. Goringe, E. Hernández, M. J. Gillan and I. J. Bush, Comput. Phys. Commun. 102, 1 (1997).

${ }^{20}$ R. Baer and M. Head-Gordon, Phys. Rev. Lett. 79, 3962 (1997).

${ }^{21}$ P. D. Haynes and M. C. Payne, Comput. Phys. Commun. 102, 17 (1997).

22 D. R. Bowler and M. J. Gillan, Comput. Phys. Commun. 112, 103 (1998).

${ }^{23}$ D. R. Bowler, M. Aoki, C. M. Goringe, A. P. Horsfield and D. G. Pettifor, Modell. Simul. Mat. Sci. Eng. 5, 199 (1997).

${ }^{24}$ R. McWeeny, Rev. Mod. Phys. 32, 335 (1960).

${ }^{25}$ P. D. Haynes and M. C. Payne, Sol. Stat. Commun. 108, 737 (1998) and submitted to Phys. Rev. Lett. (1998).

${ }^{26}$ A.H.R. Palser and D.E. Manolopoulos, Phys. Rev. B, 58, 12704.

27 C. M. Goringe, D. Phil Thesis, Oxford University (1995).

${ }^{28}$ S.-Y. Qiu, C. Z. Wang, K. M. Ho and C. T. Chan, J. Phys.: Condens. Matter 6, 9153 (1994).

${ }^{29}$ D. J. Chadi, J. Vac. Sci. Technol. 16, 1290 (1979).

${ }^{30}$ D. R. Bowler, M. Fearn, C. M. Goringe, A. P. Horsfield and D. G. Pettifor, J. Phys.: Condens. Matter 10, 3719 (1998).

31 A. P. Horsfield, P. D. Godwin, D. G. Pettifor and A. P. Sutton, Phys. Rev. B 54, 15773 (1996).

32 This is discussed for liquid carbon in Ref. 28. In our tests on liquid silicon, we found that the hybrid method, LNV with re-initialisation of $\rho$ to $\frac{1}{2} \mathbf{I}$ and LNV using the previous iteration's value of $\rho$ as initialisation all produced the same results providing they were run at a sufficiently high tolerance. The tolerance required for LNV reusing the previous value of $\rho$ was two orders of magnitude higher than that required for LNV using $\frac{1}{2} \mathbf{I}$ or the hybrid method; the iterations required were 30 for LNV using the previous $\rho$, 26 for LNV reinitialising to $\frac{1}{2} \mathbf{I}$, and 13 for each phase of the hybrid method.

${ }^{33}$ Our tests find that Hotelling's method (described in W.H.Press, S.A.Teukolsky, W.T.Vetterling and B.P.Flannery, Numerical Recipes (Cambridge University Press, Cambridge, 1992)) is extremely efficient and easily implemented, and scales linearly with system size when a cutoff is applied to $S^{-1}$. Even with a modest cutoff, the approximate inverse is extremely good.

${ }^{34}$ R. W. Nunes and D. Vanderbilt, Phys. Rev. B 50, 17622 (1994).

${ }^{35}$ C. A. White, P. Maslen, M. S. Lee and M. Head-Gordon, Chem. Phys. Lett. 276, 133 (1997).

\section{APPENDIX A: PROOF OF THE TWO STATEMENTS}

We want to prove the two statements enunciated in Sec. III: (1) all McWeeny paths meet the idempotency surface orthogonally; (2) for any point on the idempo- 
tency surface, the gradient of the LNV function is tangential to the surface.

We show first how to characterise displacements within and perpendicular to the tangent plane. Suppose $P$ is a point on the idempotency surface and let $Q=I-P$, so that $P^{2}=P$ and $Q^{2}=Q$. Consider displacements away from $P$ represented by $P^{\prime}=P+\alpha \delta P$. Any displacement $\delta P$ can be written as

$$
\delta P=\delta P_{\|}+\delta P_{\perp},
$$

where

$$
\begin{gathered}
\delta P_{\|}=P \delta P Q+Q \delta P P \\
\delta P_{\perp}=P \delta P P+Q \delta P Q
\end{gathered}
$$

It is straightforward to show that any displacement of the form $P^{\prime}=P+\alpha \delta P_{\|}$is in the tangent plane:

$$
\begin{aligned}
{P^{\prime}}^{2} & =P^{2}+\alpha\left(P \delta P_{\|}+\delta P_{\|} P\right)+\alpha^{2} \delta P_{\|}^{2} \\
& =P^{\prime}+\alpha^{2} \delta P_{\|}^{2} .
\end{aligned}
$$

On the other hand, no displacement of the form $P^{\prime}=P+$ $\alpha \delta P_{\perp}$ can be in the tangent plane, since a little algebra shows that

$$
P^{\prime 2}=P^{\prime}+\alpha(P \delta P P-Q \delta P Q)+\alpha^{2} \delta P_{\perp}^{2} .
$$

But the term linear in $\alpha$ cannot vanish unless $P \delta P P$ and $Q \delta P Q$ vanish separately, which implies that $\delta P_{\perp}=0$. It follows that the displaced point $P^{\prime}=P+\alpha\left(\delta P_{\|}+\delta P_{\perp}\right)$ is in the tangent plane if and only if $\delta P_{\perp}=0$.

Now let $P$ be a point on the idempotency surface, and let $P+\alpha \delta P$ be a point on a McWeeny path that leads to $P$. Consider the matrix $P^{\prime}$ obtained by performing a single purification on $P+\alpha \delta P$ :

$$
P^{\prime}=3(P+\alpha \delta P)^{2}-2(P+\alpha \delta P)^{3} .
$$

Because of the property of quadratic convergence, $P^{\prime}$ must deviate from $P$ only by a term of order $\alpha^{2}$. The condition that the linear term in $P^{\prime}$ vanishes is:

$$
\delta P P-2 P \delta P P+P \delta P=0 .
$$

If we decompose $\delta P$ into its tangential and perpendicular components by writing $\delta P=\delta P_{\|}+\delta P_{\perp}$, then this condition becomes:

$$
\delta P_{\|}=0
$$

The implication is that any McWeeny path leading to $P$ must approach along a direction of the form $\delta P_{\perp}$. Since $\left(\delta P_{\|}, \delta P_{\perp}\right)=0$, this proves that all McWeeny paths meet the idempotency surface at right angles to the surface.

We now prove that the gradient of the LNV function of eqn (12) is tangential to the surface. We define the elements $F_{i j}$ of this (negative) gradient as:

$$
F_{i j}=-\partial \Omega / \partial \tilde{\rho}_{j i},
$$

so that from the definition given in eqn (12) we have:

$$
F_{i j}=3\left(H^{\prime} \tilde{\rho}+\tilde{\rho} H^{\prime}\right)_{i j}-2\left(\tilde{\rho}^{2} H^{\prime}+\tilde{\rho} H^{\prime} \tilde{\rho}+H^{\prime} \tilde{\rho}^{2}\right)_{i j},
$$

where $H^{\prime} \equiv H-\mu I$. If $\tilde{\rho}$ is a point $P$ on the idempotency surface, then:

$$
F=H^{\prime} P-2 P H^{\prime} P+P H^{\prime} .
$$

It is now straightforward to show that $P^{\prime}=P+\alpha F$ is a tangent line:

$$
\begin{aligned}
P^{\prime 2} & =P^{2}+\alpha(P F+F P)+\alpha^{2} F^{2} \\
& =P+\alpha\left(H^{\prime} P-2 P H^{\prime} P+P H^{\prime}\right)+\alpha^{2} F^{2} \\
& =P^{\prime}+\alpha^{2} F^{2} .
\end{aligned}
$$

\section{APPENDIX B: THE NON-ORTHONORMAL CASE}

We show here that the two statements enunciated in Sec. III remain true when we generalize to nonorthonormal basis functions. In this case, the energy eigenvalues $\epsilon_{n}$ are determined by the generalized eigenvalue equation:

$$
\sum_{j=1}^{N} \bar{H}_{i j} \bar{c}_{j n}=\epsilon_{n} \sum_{j=1}^{N} \bar{S}_{i j} \bar{c}_{j n},
$$

where $\bar{S}_{i j}$ is the overlap matrix. (Our notation gives an over-bar to quantities in the non-orthonormal case to distinguish them from the corresponding quantities in the orthonormal case.) With $N_{0}$ occupied states, the ground-state energy $E_{0}$ is then determined by:

$$
E_{0}=\min \operatorname{Tr}(\bar{H} \bar{\rho})
$$

subject to the constraints that $\bar{\rho}$ is a 'generalized projector':

$$
\bar{\rho} \bar{S} \bar{\rho}=\bar{\rho}
$$

and that $\operatorname{Tr}(\bar{S} \bar{\rho})=N_{0}$. If we work at constant chemical potential, then we minimize the grand potential $\Omega$ :

$$
\Omega_{0}=\min \operatorname{Tr}(\bar{H}-\mu \bar{S}) \bar{\rho}
$$

subject to $\bar{\rho}$ being a generalized projector. This whole scheme corresponds precisely to the orthonormal scheme through the relations:

$$
\begin{aligned}
\bar{H} & =\bar{S}^{1 / 2} H \bar{S}^{1 / 2} \\
\bar{\rho} & =\bar{S}^{-1 / 2} \rho \bar{S}^{-1 / 2} .
\end{aligned}
$$

From this correspondence, it is clear that the McWeeny purification of the density matrix $\rho$ is accomplished by iteration of the algorithm: 


$$
\rho=3 \tilde{\rho} S \tilde{\rho}-2 \tilde{\rho} S \tilde{\rho} S \tilde{\rho} .
$$

(To simplify the notation, we now drop the over-bars.) The scheme of Palser and Manolopoulo 26 can be implemented by starting from an initial density matrix given by:

$$
\rho^{(0)}=\frac{1}{2} \xi\left(\mu S^{-1}-S^{-1} H S^{-1}\right)+\frac{1}{2} S^{-1}
$$

as has already been pointed out in Ref. 26. The constant$N_{0}$ algorithm can also be recast in non-orthonormal form.

Similarly, the Nunes and Vanderbilt (NV) method 34 at constant $\mu$ consists of minimizing $\Omega$ given by:

$$
\Omega=\operatorname{Tr}(H-\mu S)(3 \tilde{\rho} S \tilde{\rho}-2 \tilde{\rho} S \tilde{\rho} S \tilde{\rho}) .
$$

This formulation of the NV method for the nonorthonormal case has already been discussed in Refs. 34,13.

The two key statements of Sec. III remain valid provided we use the appropriate metric for defining scalar products of matrices. Instead of eqn (14), we must use the definition:

$$
(A, B)=\operatorname{Tr}\left(A^{\dagger} S B S\right),
$$

which means that the matrices $A$ and $B$ are orthogonal if $\operatorname{Tr}\left(A^{\dagger} S B S\right)=0$. The 'idempotency surface' must, of course, the taken to mean the manifold of all matrices satsifying eqn (B3). The methods of Appendix A are then straightforwardly repeated to show that McWeeny paths meet the idempotency surface orthogonally.

We now turn to the LNV gradient. Naively, one might be inclined to work with the (negative) gradient $F_{i j}=$ $-\partial \Omega / \partial \tilde{\rho}_{j i}$ as defined in the orthogonal case However, as has been stressed recently by White et al. .35 , for a metric defined by eqn. (B9), it would not be tensorially correct to update the density matrix $\tilde{\rho}$ using a gradient defined in this way, since transformations of the basis make $\tilde{\rho}_{i j}$ behave as a contravariant quantity but the derivative $F_{i j}$ as a covariant quantity. Instead, one should work with the contravariant gradient $\Phi$ defined as:

$$
\Phi_{i j}=\left(S^{-1} F S^{-1}\right)_{i j} .
$$

If one were to work with the metric defined by eqn. (14), then the original gradient of $F_{i j}$ is correct (though the two statements in Section III require the metric defined by eqn. B9).

It is straightforward to show that $\Phi$ is tangential to the idempotency surface. To do this, note from eqn (B8) that the gradient $F$ of $\Omega$ is given by:

$$
\begin{aligned}
F_{i j} & =-\partial \Omega / \partial \tilde{\rho}_{j i} \\
& =3\left(S \tilde{\rho} H^{\prime}+H^{\prime} \tilde{\rho} S\right)_{i j} \\
& -2\left(S \tilde{\rho} S \tilde{\rho} H^{\prime}+S \tilde{\rho} H^{\prime} \tilde{\rho} S+H^{\prime} \tilde{\rho} S \tilde{\rho} S\right)_{i j},
\end{aligned}
$$

where $H^{\prime} \equiv H-\mu S$. If $\tilde{\rho}$ is a point $P$ on the idempotency surface, then this reduces to:

$$
F=S P H^{\prime}-2 S P H^{\prime} P S+H^{\prime} P S,
$$

and the contravariant gradient is:

$$
\Phi=P H^{\prime} S^{-1}-2 P H^{\prime} P+S^{-1} H^{\prime} P .
$$

It then follows that $P^{\prime} \equiv P+\alpha \Phi$ is in the tangent plane:

$$
\begin{aligned}
P^{\prime} S P^{\prime} & =P+\alpha(\Phi S P+P S \Phi)+\alpha^{2} \Phi S \Phi \\
& =P^{\prime}+\alpha^{2} \Phi S \Phi .
\end{aligned}
$$

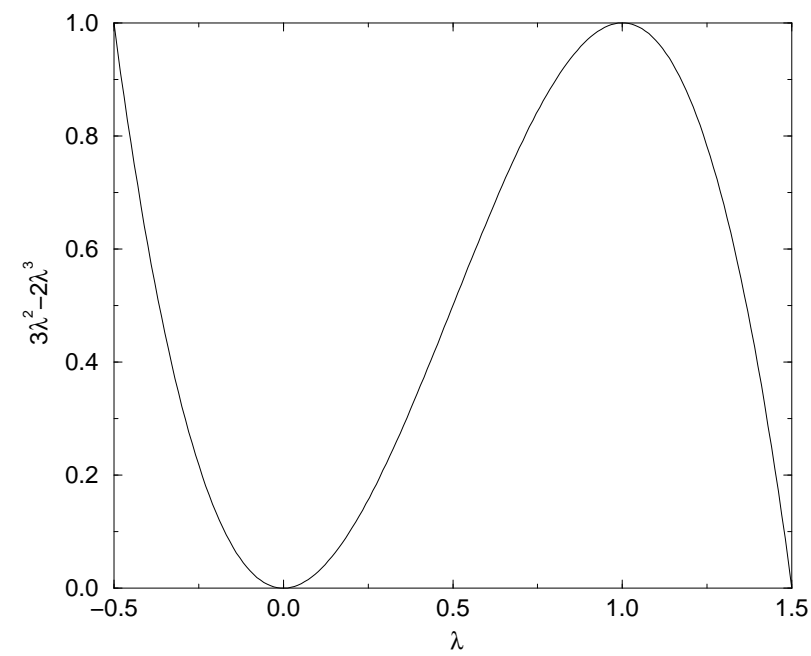

FIG. 1. The function $f(\tilde{\lambda})=3 \tilde{\lambda}^{2}-2 \tilde{\lambda}^{3}$ in the range $-\frac{1}{2}<\tilde{\lambda}<\frac{3}{2}$.

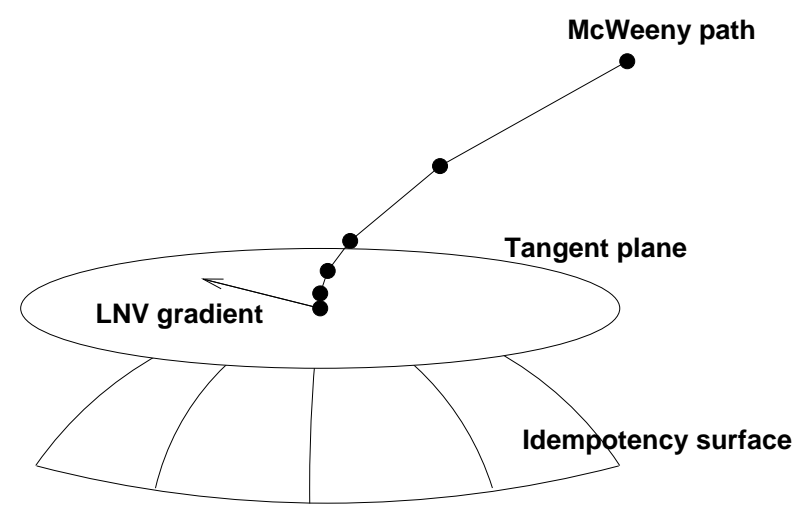

FIG. 2. Geometrical representation of the McWeeny and LNV methods, showing that the density matrices generated by McWeeny iteration form a path approaching the idempotency surface orthogonally, and that the gradient of the LNV function lies in the tangent plane to the idempotency surface. 


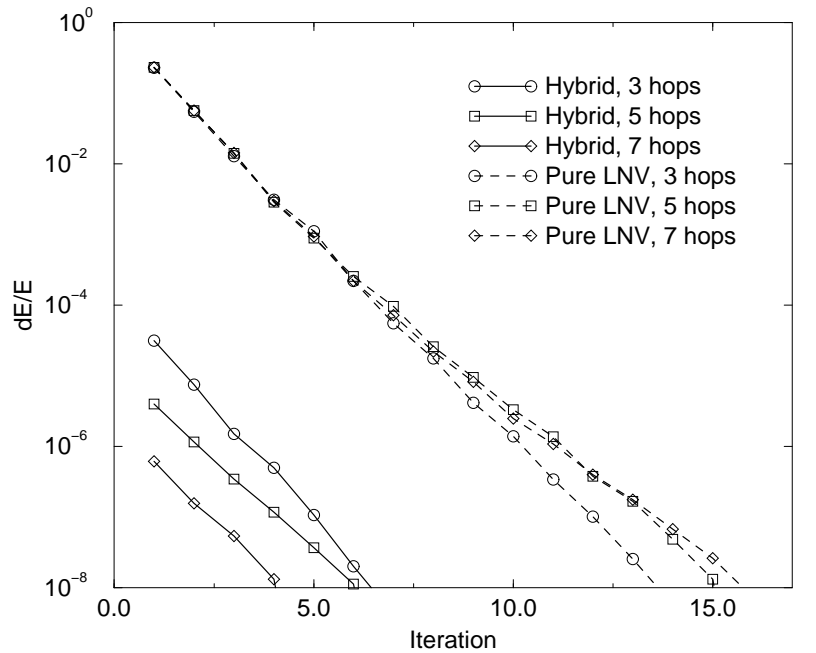

FIG. 3. The difference between the cohesive energy at a given iteration and the final cohesive energy for the LNV stage of the hybrid scheme (solid lines) and the pure LNV scheme (dashed lines) for diamond structure carbon. Results are shown for different cut-off radii: 3 hops (circles), 5 hops (squares) and 7 hops (diamonds). The radii are discussed in the text.

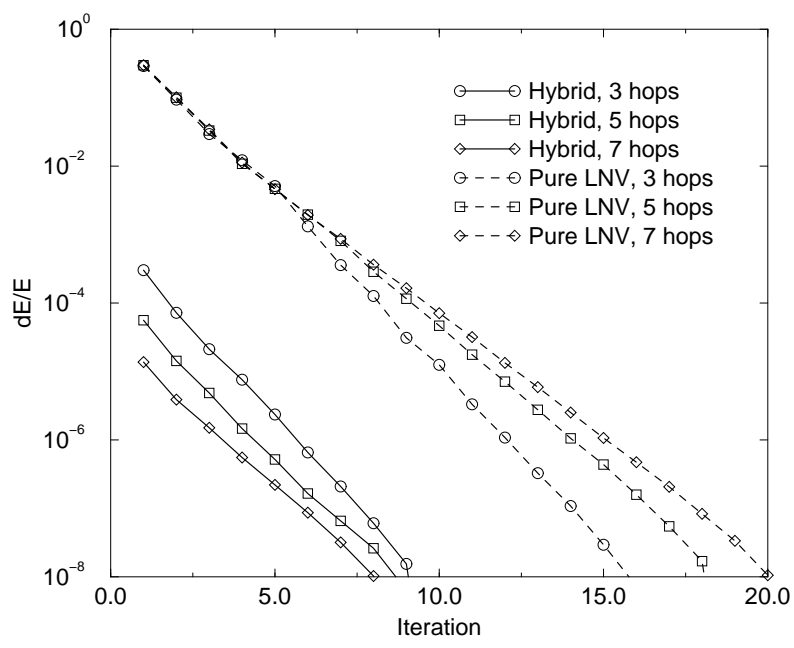

FIG. 4. The difference between the cohesive energy at a given iteration and the final cohesive energy for the LNV stage of the hybrid scheme (solid lines) and the pure LNV scheme (dashed lines) for diamond structure silicon. Results are shown for different cut-off radii: 3 hops (circles), 5 hops (squares) and 7 hops (diamonds). The radii are discussed in the text.
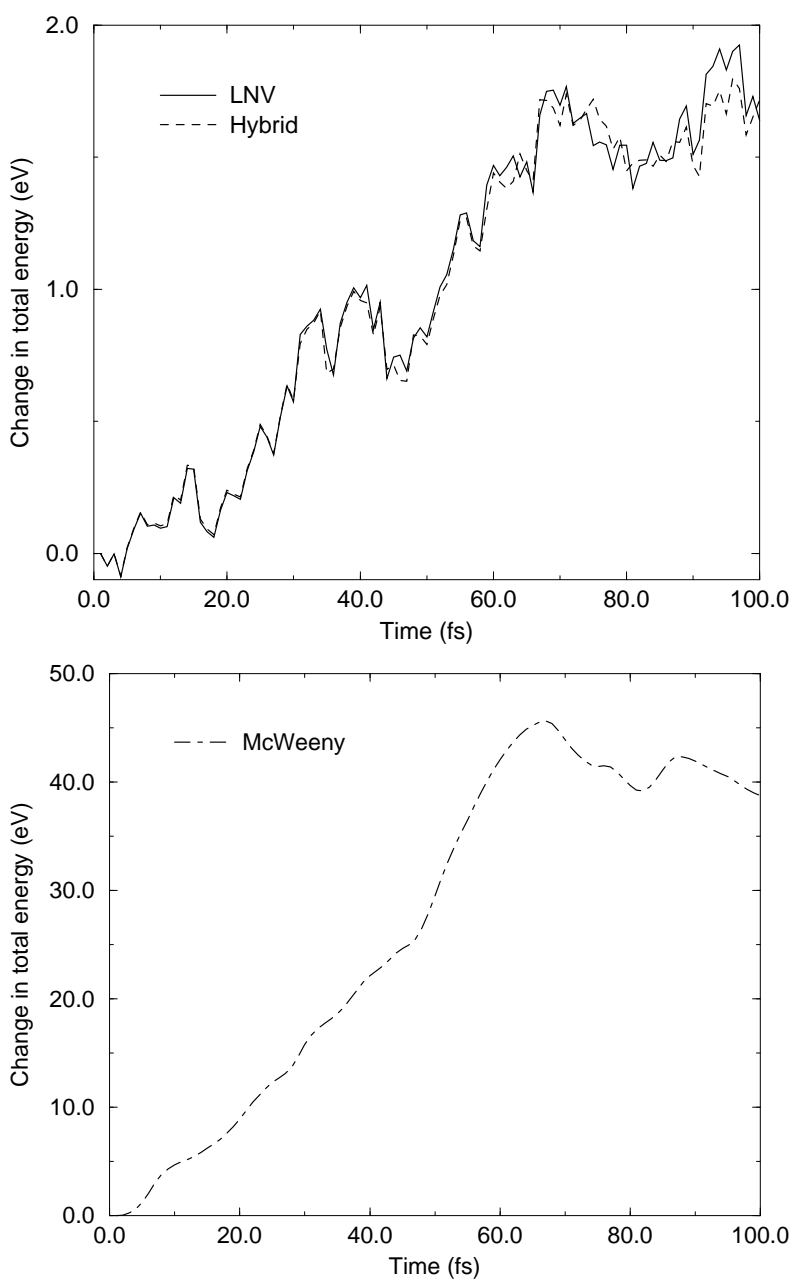

FIG. 5. Change in total energy (eV units) during molecular dynamics runs on a 64-atom system of liquid Si for (a) pure LNV (solid curve) and hybrid method (dashed curve) and (b) pure McWeeny method.

\begin{tabular}{|c|cc|c|c|}
\hline \hline Radius (hops) & \multicolumn{2}{|l}{ Iterations (Hybrid) } & Iterations (LNV) & Norm \\
& McWeeny & LNV & & \\
\hline 3 & 9 & 8 & 17 & 0.049 \\
5 & 10 & 7 & 17 & 0.015 \\
7 & 11 & 5 & 16 & 0.007 \\
\hline \hline
\end{tabular}

TABLE I. Aspects of the convergence to the ground state for the hybrid scheme and a pure LNV scheme for diamond structure carbon. The first column shows the spatial cut-off applied to the density matrix. The second and third show the number of iterations required in the McWeeny and LNV stages of the hybrid scheme, and the fourth the number of iterations required by a pure LNV scheme. The last column shows the norm of the difference between the final McWeeny density matrix and the final density matrix (see Eq. (13) in Section III for the definition of norm). The convergence criterion was a fractional difference of $10^{-8}$ between the cohesive energies in successive line minimisations. 


\begin{tabular}{|c|cc|c|c|}
\hline \hline Radius (hops) & \multicolumn{2}{|l}{ Iterations (Hybrid) } & Iterations (LNV) & Norm \\
& McWeeny & LNV & & \\
\hline 3 & 11 & 10 & 21 & 0.078 \\
5 & 12 & 10 & 21 & 0.043 \\
7 & 13 & 9 & 22 & 0.024 \\
\hline \hline
\end{tabular}

TABLE II. Aspects of the convergence to the ground state for the hybrid scheme and a pure LNV scheme for diamond

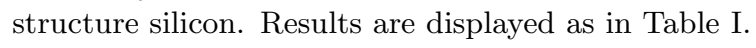

\begin{tabular}{|c|cc|c|c|}
\hline \hline Radius (hops) & \multicolumn{2}{|c|}{ Iterations (Hybrid) } & Iterations (LNV) & $\Delta N_{o}$ \\
& McWeeny & LNV & & \\
\hline 3 & 10 & 9 & 16 & 0.015 \\
5 & 11 & 8 & 18 & 0.0006 \\
7 & 12 & 6 & 18 & 0.0004 \\
\hline \hline
\end{tabular}

TABLE III. Aspects of the convergence to the ground state for the hybrid scheme and a pure LNV scheme for a relaxed vacancy in carbon. The first four columns are as in Table $\mathbb{I}$, while the last column shows the deviation, $\Delta N_{o}$, from the total electron number (252) after the LNV stage of the hybrid minimisation.

\begin{tabular}{|c|cc|c|c|}
\hline \hline Radius (hops) & \multicolumn{2}{|c|}{ Iterations (Hybrid) } & Iterations (LNV) & $\Delta N_{o}$ \\
& McWeeny & LNV & & \\
\hline 3 & 15 & 14 & 73 & 0.0311 \\
5 & 16 & 13 & 79 & 0.0213 \\
7 & 17 & 10 & 82 & 0.0221 \\
\hline \hline
\end{tabular}

TABLE IV. Aspects of the convergence to the ground state for the hybrid scheme and a pure LNV scheme for the $\mathrm{Si}(001)$ surface. The first four columns are as in Table If, while the last column shows the deviation, $\Delta N_{o}$, from the total electron number (168) after the LNV stage of the hybrid minimisation. 